\title{
Dynamically Configurable Nonlinear Optical Switching Based on Vertically Aligned Carbon Nanotubes
}

\author{
Cecilia Mercado-Zúñiga ${ }^{a}$, Carlos Torres-Torres ${ }^{b *}$, María Teresa Torres-Mancera ${ }^{a}$, Jorge Roberto \\ Vargas-García ${ }^{c}$, Reydezel Torres-Martínez ${ }^{d}$
}

\author{
${ }^{a}$ Subdirección C, Tecnológico de Estudios Superiores de Coacalco - TESCo, 55700, Coacalco de \\ Berriozábal, México, México
}

${ }^{b}$ Sección de Estudios de Posgrado e Investigación, Escuela Superior de Ingeniería Mecánica y Eléctrica

Unidad Zacatenco, Instituto Politécnico Nacional, 07738, México, Distrito Federal, México

'Depto. Ing. Metalurgia y Materiales, Escuela Superior de Ingenieria Quimica e Industrias Extractivas

- ESIQIE, Instituto Politécnico Nacional - IPN, 07300, México, DF, México

${ }^{d}$ Centro de Investigación en Ciencia Aplicada y Tecnología Avanzada Unidad Querétaro, Instituto

Politécnico Nacional, 76090, Santiago de Querétaro, Querétaro, México

Received: January 21, 2016; Revised: June 3, 2016; Accepted: July 15, 2016

\begin{abstract}
The modification of the third order nonlinear optical response exhibited by vertically aligned carbon nanotubes in two-wave mixing interactions was analyzed. All-optical switching effects were explored by using a vectorial optical Kerr gate with nanosecond pulses at $532 \mathrm{~nm}$ wavelength of excitation. The samples were prepared by a spray pyrolysis method. Morphological and structural characterization of the samples was conducted by Scanning Electron Microscopy, Transmission Electron Microscopy and Raman Spectroscopy studies. Important structural changes dependent on the temperature employed for the preparation of the carbon nanotubes were observed. It was highlighted a remarkable influence of nonlinear energy transference in spatially-resolved optical processes for designing ultrafast lowdimensional devices.
\end{abstract}

Keywords: Nonlinear Optics, Two-wave mixing, aligned carbon nanotubes

\section{Introduction}

Carbon nanostructures have attracted the attention of numerous researchers in regards to their fascinating mechanical, optical and electronic characteristics. It is well-known that the extraordinary physical behavior of carbon nanomaterials concern to their morphology, structure and growth mechanism. ${ }^{1,2}$ Besides, the manufacturing of nanohybrids based on tailored nanoparticle interactions with carbon nanosystems has been extensively considered for diverse multi-functional applications. ${ }^{3,4}$ Particularly, the one-dimensional character and chirality of carbon nanotubes (CNTs) give origin to a significant number of unusual optical phenomena such as optical activity, circular dichroism, and second-harmonic generation. ${ }^{5}$ Third order optical nonlinearities, like the optical Kerr effect and two-photon absorption, take a relevant importance due to the possibility to modulate optical signals by light. An ultrafast control of information can be conceived by multi-wave coupling of optical signals governed by the third order nonlinear optical response. Moreover, the fabrication of nanoelectronic devices can take advantages from positioning individual or self-assembled nanofibers on particular spots with nanometric resolution. ${ }^{6}$ In this direction, the procedure to obtain aligned arrays of

* e-mail: crstorres@yahoo.com.mx; ctorrest@ipn.mx
CNTs seems to be useful to contribute on the manipulation of properties and possible nanophotonic applications.

It has been pointed out that the spray pyrolysis mechanisms for preparing CNTs are not currently fully understood, ${ }^{7-8}$ however, a the strong dependence of the CNTs characteristics on their growth mechanism has been reported. ${ }^{9}$

Although there are several techniques for obtaining carbon nanotubes, ${ }^{10}$ the spray pyrolysis method has shown significant advances in the production of nanostructures; regarding that this approach can be scalable to the industrial level. ${ }^{11}$ Advantages such as low-cost and easy handling, make the spray pyrolysis technique to be a good candidate for developing carbon-based nanostructures; for instance aligned carbon nanotubes (ACNTs). In general, ACNTs exhibit a very large surface area and a high thermal conductivity; these characteristics allow a fast energy transfer, which is definitively useful for ultrafast nanodevices. ${ }^{12}$

The enhancement of the optical and photoconductive response associated with the orientation of CNTs has been distinguished for regulation of electronic and multi-photonic phenomena. ${ }^{13}$ In particular, randomly distributed networks of CNTs present an inhomogeneous electronic behavior that may be improved by hierarchical distributions. ${ }^{14}$ Undoubtedly, ACNTs are suitable for maximizing access to specific surfaces in nanoelectronic systems construction. ${ }^{15}$ 
Furthermore, outstanding applications for free-propagation electromagnetic waves transduction have been achieved by using vertically ACNTs. ${ }^{16}$

Several investigations have been conducted towards obtaining ACNTs with specific optical and electronic characteristics. Some examples that are based on the pyrolysis of mixtures rich in carbon precursors and metal catalysts, promote the growth of extraordinary nanostructures so-called mixtures of green carbon precursors. ${ }^{17-18}$ It is worth noting that the use of ferrocene for preparing carbon nanostructures allows the growth of densely packed ACNTs, with several microns in length. However, in some cases, the ACNTs can constitute bundles of aligned shaped structures, due to the nitrogen incorporation. ${ }^{19} \mathrm{CNTs}$ acquired by a final scraping in the quartz tube where were prepared, result in an unpredictable performance dependent on the final length and morphology of some tubes..$^{20}$ One way to potentiate the ACNTs applications could be formulating a growth of the tubes on the desired substrate.

In this regard, a substantial contribution of the surrounding of nanostructures can derive in the enhancement of their nonlinear optical parameters. It is noteworthy that particular substrates for supporting CNTs can be suitable to provide lab-on-a-chip applications. Various materials such as clays, metals in nanometric size and other nanocomposites with different characteristics have been used as substrates for growing CNTs. ${ }^{21-23}$ However, the synergy between Zirconia $\left(\mathrm{ZrO}_{2}\right)$ and CNTs has proved to be attractive for developing biocompatible and electrochemically superior biosensors. With these considerations, $\mathrm{ZrO}_{2}$ substrates seem to be an interesting platform for catalyst and instrumentation applications as it has been previously suggested. ${ }^{24}$ Moreover, $\mathrm{ZrO}_{2}$ can be considered a nonmagnetic material with high melting temperature and relatively inert to oxidation media for growing CNTs to develop possible optical and electronic functions. ${ }^{25}$ Then, with this motivation, within this work the third order nonlinear optical characteristics exhibited by vertically ACNTs grown on $\mathrm{ZrO}_{2}$ substrates were evaluated. Our results indicated that nanostructured periodicity and hierarchical energy transference can be responsible for amplification or inhibition of nonlinear optical effects.

\section{Experimental}

Polycrystalline $\mathrm{ZrO}_{2}$ wafers (5 $\mathrm{mm}$ of diameter) were used as substrates to grow CNTs. The substrates were located in two different heating zones (zone $1=800{ }^{\circ} \mathrm{C}$ and zone $2=900{ }^{\circ} \mathrm{C}$ ), inside of tubular hot-wall reactor, according to Figure 1. A ferrocene/toluene mixture (3.5/96.5 wt \%; ferrocene: Sigma Aldrich, toluene: Fermont $99.9 \%$ purity) was supplied as Fe and C. Argon (Infra 99.999\% purity) was employed as the carrier gas with a flow rate of $2.5 \mathrm{l} / \mathrm{min}$. The reactor was operated with $25 \mathrm{~mm}$ of $\mathrm{H}_{2} \mathrm{O}$ pressure $(0.24$ $\mathrm{kPa})$ above atmospheric pressure $(\sim 80 \mathrm{kPa})$ to prevent any

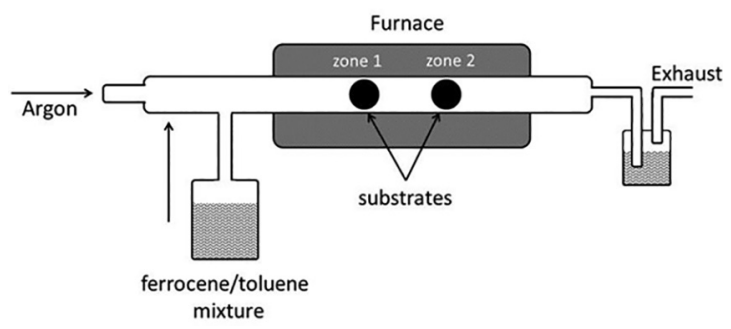

Figure 1: Schematic illustration of the experimental setup used to grow CNTs on Zirconia substrates.

influx of oxygen. After $16 \mathrm{~min}$ of reaction, the system was cooled down to reach room temperature for removing the resulting $\mathrm{ZrO}_{2}$ wafer substrates.

In order to investigate the nanotubes structure, Raman spectroscopy studies were undertaken. The measurements were carried out by using a Jobin Yvon Horiba HR 800 with $633 \mathrm{~nm}$ laser in the range $100-3100 \mathrm{~cm}^{-1}$. CNTs morphology was observed by Scanning Electron Microscopy and confirmed by Transmission Electron Microscopy (FESEM; JEOL JSM-6701F, TEM; JEOL JEM-2200FS). The role of third order nonlinear optical behavior of the samples was explored by using a vectorial Optical Kerr Gate (OKG) in a reflective configuration. ${ }^{26} \mathrm{~A} \mathrm{Nd:YAG} \mathrm{system} \mathrm{Continuum}$ Model SL II-10 was used as a laser source featuring 4 nanoseconds pulse duration at $532 \mathrm{~nm}$ wavelength and $1 \mathrm{~mJ}$ of average energy. Two incident beams, pump and probe, with irradiance relation 1:1 were impinging into the sample with linear polarizations and a beam diameter close to $1 \mathrm{~mm}$. The plane of polarization of the probe beam was varied by a half-wave plate in order to confirm the vectorial nature of the nonlinear optical behavior of the interaction. The experimental setup was previously calibrated using a silicon sample as a nonlinear medium with a well-known third-order nonlinear susceptibility of $\left|\chi^{(3)}\right|=2.0 \times 10^{10} \mathrm{esu}^{27}$

\section{Results and Discussions}

Raman spectra of CNTs of the sample at two different temperatures are shown in Figure 2. Raman spectra revealed three bands around 1338, 1588 and $2659 \mathrm{~cm}^{-1}$, which are attributed to D (associated to impurities and defects), G (graphitic nature), and $\mathrm{G}^{\prime}$ (second order of graphitic structure) bands, at the same positions for two different synthesis conditions; respectively. Raman spectrum of CNTs prepared at $800{ }^{\circ} \mathrm{C}$ presented a high intensity background; this behavior is frequently associated to fluorescence phenomena of the sample itself. A group of bands on values $\leq 200 \mathrm{~cm}^{-1}$ probably related to single walled CNTs (RBM band) can be also observed. Additionally, a group of small peaks are clearly visible between 200 and $400 \mathrm{~cm}^{-1}$, which may suggest the presence of metallic particles. A way to know the crystal 


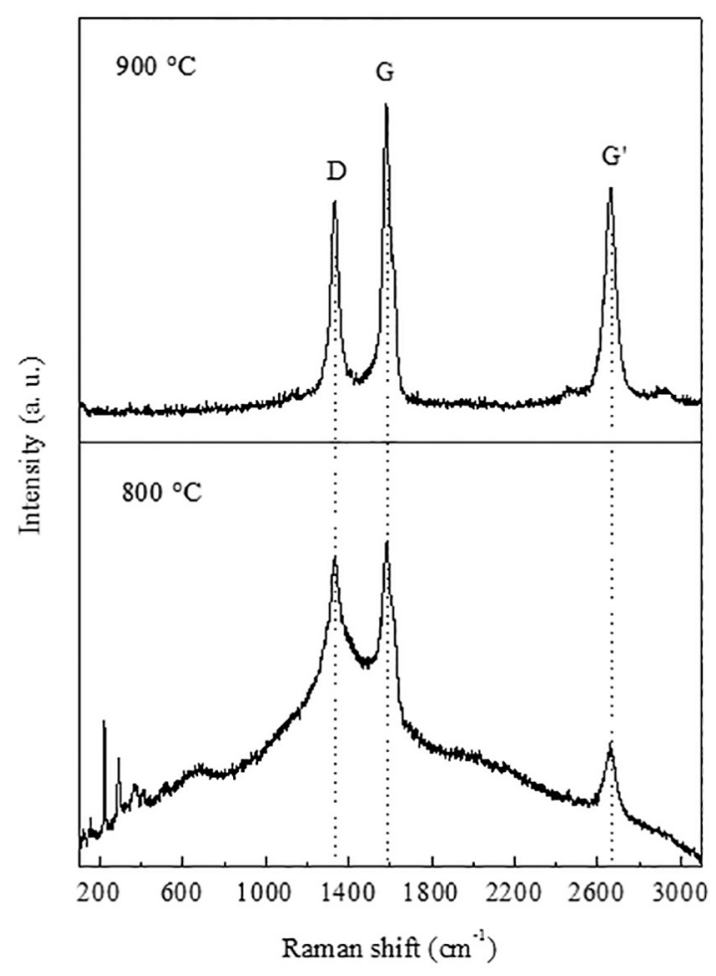

Figure 2: Raman spectra of CNTs growth on Zirconia wafers substrates at two different temperatures.

quality of CNTs is by considering the relative intensity ratio of $D$ and $G$ bands $\left(I_{D} / I_{G}\right)$. As a result of this calculation in our cases, was obtained 0.97 and 0.68 for CNTs grown at $800^{\circ} \mathrm{C}$ and $900^{\circ} \mathrm{C}$, respectively. It can be expected for the $800^{\circ} \mathrm{C}$ case, a lot of amorphous carbon while the CNTs grown at $900{ }^{\circ} \mathrm{C}$ presented a better graphitization degree. The higher intensity on the $\mathrm{G}^{\prime}$ band in the spectrum at 900 ${ }^{\circ} \mathrm{C}$ is consistent with this result.

SEM images related to CNTs grown at 800 and $900{ }^{\circ} \mathrm{C}$ on $\mathrm{ZrO}_{2}$ substrates are shown in Figure 3. The images showed ACNTs growing normal to $\mathrm{ZrO}_{2}$ substrate surface forming a kind of "carpet" in both cases, however, particularly at 800 ${ }^{\circ} \mathrm{C}$ (a) can be observed a bright area at the tip of the ACNTs. Elemental analysis on ACNTs near at tip regions indicates the presence of $\mathrm{Fe}$, which can be associated with the presence of catalyst particles remaining from the process of nanotubes growth, in accordance with Raman results. The thickness of the ACNTs carpet was approximately $10 \mu \mathrm{m}$ for the two different temperatures studied. It is worth to mention that the "carpet" of ACNTs can be easily peeled off from the $\mathrm{ZrO}_{2}$ substrate without destroying its integrity and therefore, the ACNTs grown at $900{ }^{\circ} \mathrm{C}$ was selected for subsequent analysis. The evaluation of the single- and multi-wall nature exhibited by these samples was quantified according to the Raman band intensities. ${ }^{28}$ Our estimations indicate that a content of about $10 \mathrm{wt} \%$ for single-wall CNTs and $90 \mathrm{wt} \%$ for multi-wall CNTs correspond to the studied samples.
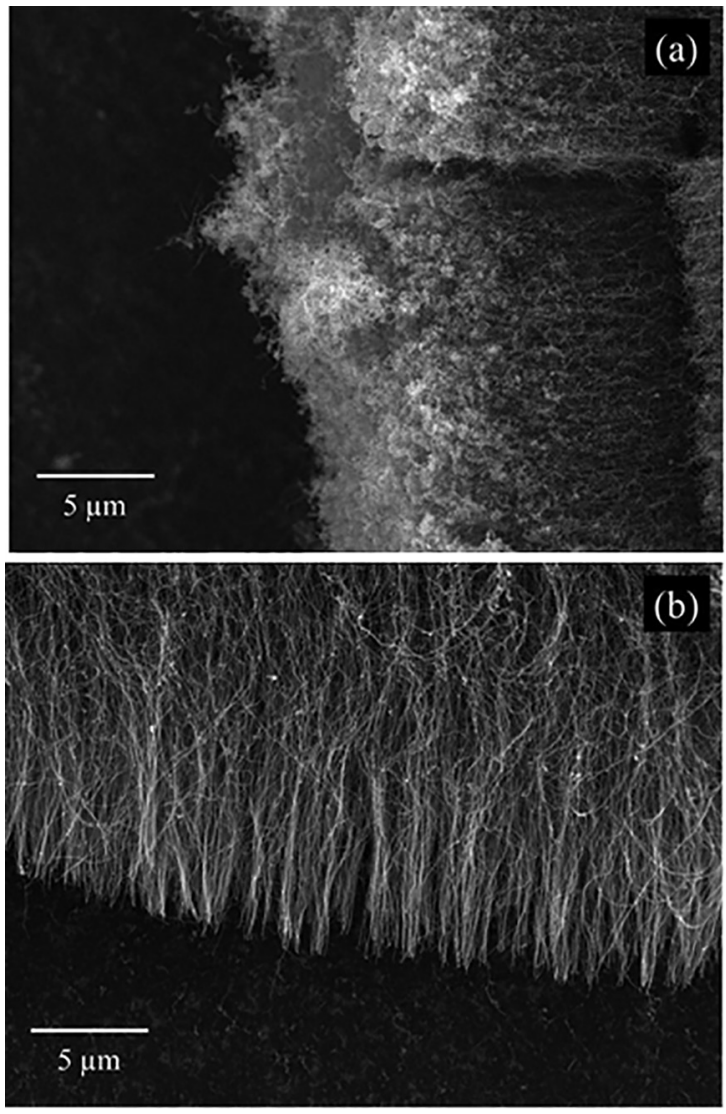

Figure 3: SEM images of CNTs grown at 800 and $900{ }^{\circ} \mathrm{C}$ on Zirconia substrates.

A TEM image of ACNTs at $900^{\circ} \mathrm{C}$ is exhibited in Figure 4. The presence of catalytic particles encapsulated in the tip and along of the nanotubes is clearly observed. These characteristics are associated with the growth mechanism like tip. ${ }^{29,30}$ The particles inside of the nanotubes seem to cause a variation in their diameter according to the particle position of nanotube to finally originate a bamboo-like appearance. ACNTs diameter was between $70 \mathrm{~nm}$ and 120 $\mathrm{nm}$ according to the observations made by TEM studies. The nanotube growth begins after $\mathrm{Fe}$ incorporation, from ferrocene precursor, deposited on the substrate as fine particles. The growth of the nanotubes is normal to the $\mathrm{ZrO}_{2}$ surface; this effect happens when carbon is deposited on metal particle's surface and diffuses through and over the metal to precipitate in the opposite face. While the carbon diffusion from toluene precursor evolves, the nanotubes continue growing and some catalyst particles become trapped at the tip or along the nanotubes, which give rise to variations in the diameter of the nanotube.

On the other hand, nonlinear optics generally concerns to the study of intense laser light interacting with matter. The presence of high optical irradiances may modify the optical parameters of a material. Certainly, nonlinear optical interactions cause that the optical response of materials present 


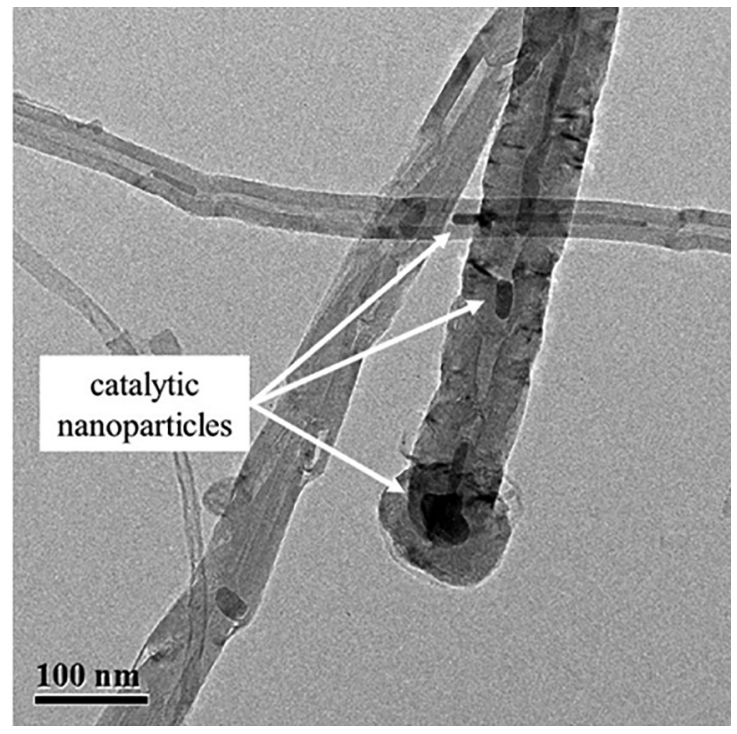

Figure 4: TEM image of CNTs grown on a Zirconia substrate.

a nonlinear dependence on the applied optical field. In this way, third order nonlinear optical effects emerge as a part of the atomic response of the media that cubically depend on the strength of the applied optical field. The optical response can be expressed as a power series by the relation between the polarization, $\mathrm{P}$, and electrical field, E, as: ${ }^{27}$

$$
P=\chi^{(1)} E+\chi^{(2)}+E \chi^{(3)} E+\ldots
$$

with $\chi^{(2)}$ and $\chi^{(3)}$ the second- and third-order nonlinear optical susceptibilities. Since the third order nonlinear optical response influences the absorption and refraction of a media for high irradiance interactions, the third order optical nonlinearities are useful for controlling light by light. The phase of an optical beam can be modified by the optical Kerr effect, which is a refractive index dependent on intensity. By contrast, the magnitude of the optical irradiance can be self-regulated by multi-photonic absorption effects; which are ruled by the imaginary part of the third order nonlinear optical susceptibility.

The evolution of nano-fabrication technologies has indicated that the periodic nanostructures in photonics facilitate a far-reaching control of light propagation and light-matter interaction. ${ }^{31}$ Nevertheless, the enhancement of some electromagnetic field effects has been contemplated to be carried out by designing deterministic aperiodic systems. ${ }^{32}$ Particularly, the alignment of low-dimensional carbon nanostructures generates the increase of transition probabilities that may be useful for developing polarizableselectable devices. ${ }^{33}$ So, for further investigate the relationship of periodic nanostructures interacting with optical signals, we modeled the third order nonlinear optical response of ACNTs in a two-wave mixing interaction. Taking into account the considerations related to mechanical and nonlinear phenomena in carbon-based nanohybrid systems, ${ }^{34}$ we used the mathematical expressions for describing the superposition of the electrical fields in the sample studied:

$$
\begin{gathered}
\Psi_{ \pm}^{(0)}=\frac{4 \pi^{2} z}{n_{0} \lambda}\left[\frac{n_{0} \beta}{2 \pi} \sum_{j-1}^{4}\left|E_{j \pm}+E_{j \mp}\right|^{2}\right], \\
\Psi_{ \pm}^{(1)}=\frac{4 \pi^{2} z}{n_{0} \lambda}\left[\frac{n_{0} \beta}{2 \pi} \sum_{j-1}^{3} \sum_{k-2}^{4}\left(E_{j \pm} E_{k \pm}^{*}+E_{j \mp} E_{k \mp}^{*}\right)\right]
\end{gathered}
$$

as the nonlinear phase changes. Here $\beta$ represents the nonlinear optical absorption coefficient, $\lambda$ is the optical wavelength, $n_{0}$ is the index of refraction, $\mathrm{z}$ is the length of the sample, and the electric fields are:

$$
\begin{gathered}
E_{1 \pm}(z)=\left[\begin{array}{l}
E_{1 \pm}^{0} J_{0}\left(\Psi_{ \pm}^{(1)}\right)+\left(i E_{2 \pm}^{0}-i E_{3 \pm}^{0}\right) \\
J_{1}\left(\Psi_{ \pm}^{(1)}\right)-E_{4 \pm}^{0} J_{2}\left(\Psi_{ \pm}^{(1)}\right)
\end{array}\right] \exp \left(-i \Psi_{ \pm}^{(0)}-\frac{\alpha(1) z}{2}\right), \\
E_{2 \pm}(z)=\left[\begin{array}{l}
E_{2 \pm}^{0} J_{0}\left(\Psi_{ \pm}^{(1)}\right)+\left(i E_{4 \pm}^{0}-i E_{i \pm}^{0}\right) \\
J_{1}\left(\Psi_{ \pm}^{(1)}\right)-E_{3 \pm}^{0} J_{2}\left(\Psi_{ \pm}^{(1)}\right)
\end{array}\right] \exp \left(-i \Psi_{ \pm}^{(0)}-\frac{\alpha(1) z}{2}\right),
\end{gathered}
$$

where $E_{1 \pm}(z)$ and $E_{2 \pm}(z)$ are the complex amplitudes of the circular components of the transmitted wave beams; $E_{3 \pm}(z)$ and $E_{4 \pm}(z)$ are the amplitudes of the self-diffracted waves, while $E_{1 \pm}^{0}, E_{2 \pm}^{0}, E_{3 \pm}^{0}$ and $E_{4 \pm}^{0}$ are the amplitudes of the incident and self-diffracted waves at the surface of the sample; $I$ is the total irradiance of the incident beams and $J_{m}\left(\psi_{ \pm}^{(1)}\right)$ stands for the Bessel function of order $m$.

Eventually, it can be expected that different nonlinear optical behaviors must be occurring for distinct angles associated with the geometry of the structures; besides, the modulation of the irradiance fringe pattern originated by a two-wave mixing is also an important contribution for the generation of an induced nonlinearity in this scheme. In fact, diffraction by an induced grating of absorption and interference of waves are the main responsible effects for the coupling of the electromagnetic fields in the sample considered. Therefore, the relative proportion between dimensions of the aligned tubes and the geometry of the interacting optical beams is a strong influence on the optical nonlinearities that originate the energy transference mechanisms that evolve by changing geometric conditions.

Changes in polarization of the probe beam in interaction with the pump beam during the OKE experiments can be expected. We assumed that the absorption coefficient dependent on irradiance $\alpha(\mathrm{I})$ in a two-wave mixing process can be written:

$$
\alpha(I)=\alpha_{o}+\beta I
$$

with $\alpha_{\mathrm{o}}$ and $\beta$ corresponding to the linear and nonlinear optical coefficients. We considered that the spatial period $\mathrm{T}_{\mathrm{f}}$, resulting from the variation of the interacting geometrical angle $\theta$, with a $\lambda=532 \mathrm{~nm}$ can be described as:

$$
T_{f}=\frac{\lambda}{2 \sin \left(\frac{\theta}{2}\right)}
$$


Moreover, the spatial period of the nanostructured arrays here is represented by $\mathrm{T}_{\mathrm{NPS}}$ and the rate relation between these two periodicities, $\mathrm{T}_{\mathrm{f}} / \mathrm{T}_{\mathrm{NPS}}$ is contemplated to be varied during an exploration. Following these considerations, in Figure 5 are illustrated our calculations by using Ecs. (1-7) for the predicted nonlinear optical enhancement in the probe beam transmittance emerging from a two-wave mixing.

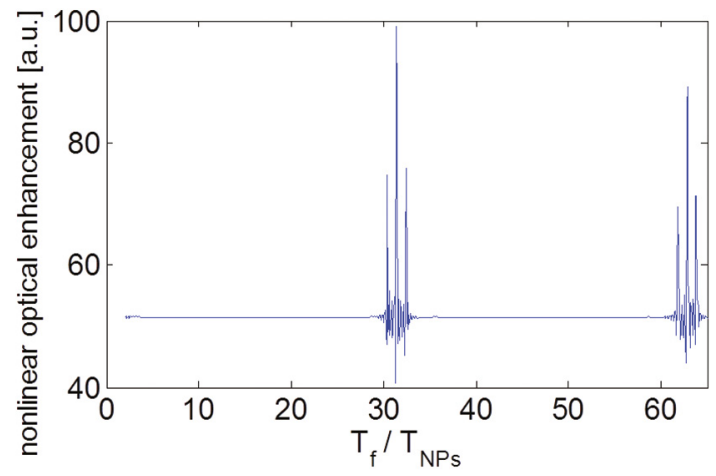

Figure 5: Third order nonlinear optical enhancement as a function of Tf/TNPs.

As it can be clearly seen from Figure 5, a sharp selective enhancement can be obtained by the modification of the spatially-resolved interaction and it can be considered to be employed for configurable switching applications.

For further investigate the nanosecond third order nonlinear optical response of ACNTs, a vectorial OKG was implemented. Different experiments performed in similar laboratory conditions were undertaken in order to report Figure 6. Each point in the experimental plot represents an average of 10 measurements. Deviation of numerical and experimental data from Figure 6 mainly corresponds to third order nonlinearities. Then, the error in the experimental data of the experiment exhibits a cubic dependence in respect to the variation of the incident irradiance; this value is around $\pm 18 \%$ in our measurements. Moreover, we noticed a strong participation related to a scattering effect together to the nonlinear optical effect in the two-wave mixing studied in the sample. This situation seems to be responsible for the linear behavior at the 10-35 degree of angle of polarization, where it is expected that the contribution of the nonlinear phenomena can be weaker than the scattering effect. Different probe transmittances were found in the samples as it can be clearly seen from the plot shown in Figure 6. Considering a purely nonlinear optical absorptive effect in the sample, it can be deduced from these records that the estimated twophoton absorption coefficient for the ACNTs was about $\beta$ $=1 \times 10^{-10} \mathrm{~m} / \mathrm{W}$. Similar parameters have been previously reported for comparative CNTs. ${ }^{35,36}$

According to the calculations illustrated in Figures 5 and 6 , the correspondent enhancement in the nonlinear optical absorption in vertically ACNTs can be observed in Table 1.

It is remarkable that the coordination of the singular and collective responses exhibited by ACNTs seems to give rise to a strong modification in the optical response of a thin film. We estimate that the efficiency of all-optical communication devices that work with multiphotonic excitations can be improved with an accurate design of the constitutive elements related to nanostructured samples. Furthermore, we envisioned the option to tailor phase-matched conditions for quantum information systems based on optical nonlinearities. It can be considered that ACNTs can be good candidates for proposing all-optical nano-switching devices.

\section{Conclusions}

Numerical and experimental results about a two-wave mixing experiment performed in ACNTs were described. The reported analysis indicated that nonlinear optical absorption effects in ACNTs strongly dependent on periodicity when the sample is irradiated by the superposition of optical waves. It is stated that a modification in the third order nonlinear optical properties exhibited by ACNTs can be derived from

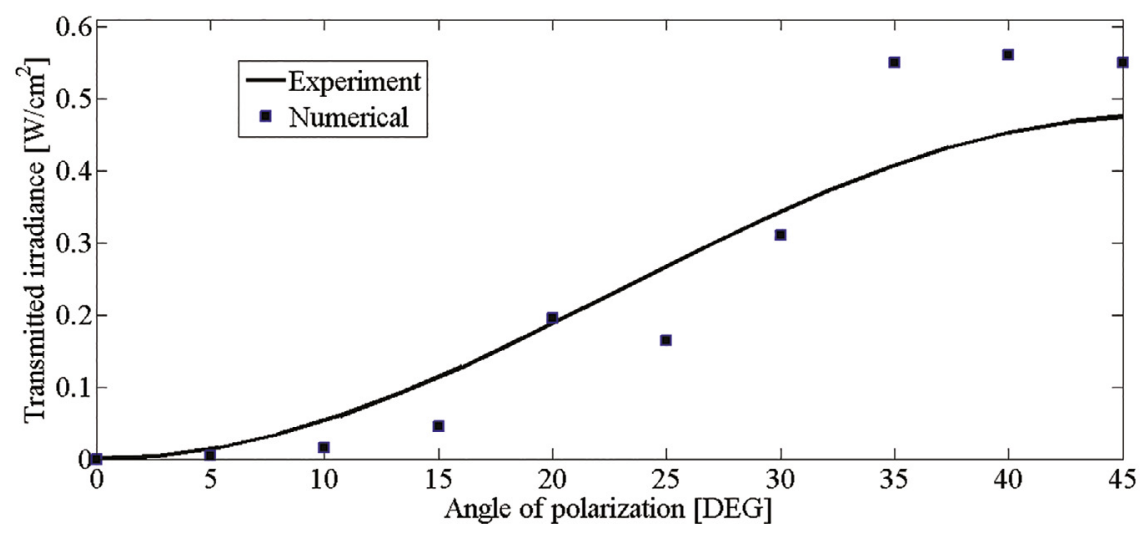

Figure 6: Nonlinear optical probe transmittance vs. angle of polarization of the interacting beams. 
Table 1: Representative variations of the nonlinear optical absorption coefficient, $\beta$, estimated for an array of vertically aligned carbon nanotubes in a two-wave mixing interaction at $532 \mathrm{~nm}$ wavelength of excitation. Here the spatial period of the fringe irradiance pattern is $\mathrm{T}_{\mathrm{f}}$, and the spatial period of the aligned carbon nanotubes is $\mathrm{T}_{\mathrm{NPS}}$

\begin{tabular}{lc}
\hline $\mathrm{T}_{\mathrm{f}} / \mathrm{T}_{\mathrm{NPS}}$ & $\beta[\mathrm{m} / \mathrm{W}]$ \\
\hline $1-30$ & $1 \times 10^{-10}$ \\
30.2 & $1.45 \times 10^{-10}$ \\
31.5 & $0.93 \times 10^{-10}$ \\
32 & $1.99 \times 10^{-10}$ \\
32.5 & $1.47 \times 10^{-10}$ \\
$33-60$ & $1 \times 10^{-10}$ \\
\hline
\end{tabular}

optical coupling and energy transference. Nonlinear optical switching devices controlled by geometric conditions of ACNTs can be contemplated.

\section{Acknowledgments}

We kindly acknowledge the financial support from Tecnológico de Estudios Superiores de Coacalco, Instituto Politécnico Nacional and Consejo Nacional de Ciencia y Tecnología (CB-243427 and CB-251201).

\section{References}

1. Andrews R, Jacques D, Rao AM, Derbyshire F, Qian D, Fan X, et al. Continuous production of aligned carbon nanotubes: a step closer to commercial realization. Chemical Physics Letters. 1999;303(5-6):467-474.

2. Musso S, Fanchini G, Tagliaferro A. Growth of vertically aligned carbon nanotubes by CVD by evaporation of carbon precursors. Diamond and Related Materials. 2005;14(3-7):784-789.

3. Scarselli M, Camilli L, Castrucci P, Nanni F, Del Globbo S, Gautron E, et al. In situ formation of noble metal nanoparticles on multiwalled carbon nanotubes and its implication in metalnanotube interaction. Carbon. 2012;50(3):875-884.

4. Wong SS, Joselevich E, Woolley AT, Cheung CL, Lieber CM. Covalently functionalized nanotubes as nanometer-sized probes in chemistry and biology. Nature. 1998;394(6688):52-55.

5. Tasaki S, Maekawa K, Yamabe T. $\pi$-band contribution to the optical properties of carbon nanotubes: Effects of chirality. Phsysical Review B. 1998;57(15):9301-9318.

6. Postma HWC, Teepen T, Yao Z, Grifoni M, Dekker C. Carbon nanotube single-electron transistors at room temperature. Science. 2001;293(5527):76-79.

7. Pinault M, Mayne-L'Hermite M, Reynaud C, Beyssac O, Rouzaud $\mathrm{JN}$, Clinard C. Carbon nanotubes produced by aerosol pyrolysis: growth mechanisms and post-annealing effects. Diamond and Related Materials. 2004;13(4-8):1266-1269.
8. Aguilar-Elguézabal A, Antúnez W, Alonso G, Paraguay-Delgado F, Espinosa F, Miki-Yoshida M. Study of carbon nanotubes synthesis by spray pyrolysis and model of growth. Diamond and Related Materials. 2006;15(9):1329-1335.

9. Lehman JH, Terrones M, Mansfield E, Hurst KE, Meunier V. Evaluating the characteristics of multiwall carbon nanotubes. Carbon. 2011;49(8):2581-2602.

10. Prasek J, Drbohlavova J, Chomoucka J, Hubalek J, Jasek O, Adam V, et al. Methods for carbon nanotubes synthesis-review. Journal of Materials Chemistry. 2011;21(40):15872-15884.

11. Sadeghian Z. Large-scale production of multi-walled carbon nanotubes by low-cost spray pyrolysis of hexane. New Carbon Materials. 2009;24(1):33-38.

12. Seah CM, Chai SP, Mohamed AR. Synthesis of aligned carbon nanotubes. Carbon. 2011;49(14):4613-4635.

13. Mercado-Zúñiga $C$, Torres-Torres $C$, Trejo-Valdez M, Torres-Martínez R, Tarrago-Velez S, Cervantes-Sodi F, et al. Mechanooptic regulation of photoconduction in functionalized carbon nanotubes decorated with platinum. International Journal of Photoenergy. 2014;2014:8p.

14. El Mel AA, Achour A, Xu W, Choi CH, Gautron E, Angleraud B, et al. Hierarchical carbon nanostructure design: ultra-long carbon nanofibers decorated with carbon nanotubes. Nanotechnology. 2011;22(43):435302.

15. He P, Dai L. Aligned carbon nanotube-DNA electrochemical sensors. Chemical Communications. 2004;7(3):348-349.

16. Sharma A, Singh V, Bougher TL, Cola BA. A carbon nanotube optical rectenna. Nature Nanotechnology. 2015;10(12):1027-1032.

17. Kumar R, Tiwari RS, Sivastava ON. Scalable synthesis of aligned carbon nanotubes bundles using green natural precursor: neem oil. Nanoscale Research Letters. 2011;6(1):92.

18. Awasthi K, Kumar R, Tiwari RS, Srivastava ON. Large scale synthesis of bundles of aligned carbon nanotubes using a natural precursor: turpentil oil. Journal of Experimental Nanoscience. 2010;5(6):498-508.

19. Kumar R, Yadav RM, Awasthi K, Tiwari RS, Srivastava ON Effect of nitrogen variation on the synthesis of vertically aligned bamboo-shaped C-N nanotubes using sunflower oil. International Journal of Nanoscience. 2011;10(4-5):809-813.

20. Torres-Torres C, Rebollo NR, Castañeda L, Trejo-Valdez M, Torres-Marínez R. Exclusive-OR encryption by photoconduction and two-photon absorption in carbon nanotubes. Journal of Nanoscience and Nanotechnology. 2015;15(2):1647-1652.

21. Angulakshmi VS, Rajasekar K, Sathiskumar C, Karthikeyan S. [1] Growth of vertically aligned carbon nanotubes on a silicon substrate by a spray pyrolysis method. New Carbon Materials. 2013;28(4):284-287.

22. Destrée A, Long GJ, Vatovez B, Grandjean F, Fonseca A, Nagy $\mathrm{JB}$, et al. Synthesis and characterization of carbon nanotubes grown on montmorillonite clays catalyst. Journal of Materials Science. 2007;42(20):8671-8689.

23. Lu FL, Ting JM. Very rapid growth of aligned carbon nanotubes on metallic substrates. Acta Materialia. 2013;61(6):2148-2153. 
24. Das M, Dhand C, Sumana G, Srivastava AK, Vijayan N, Nagarajan R, et al. Zirconia grafted carbon nanotubes based biosensor for M. Tuberculosis detection. Applied Physics Letters. 2011;99(14):143702.

25. Steiner SA 3rd, Baumann TF, Bayer BC, Blume R, Worsley MA, MoberlyChan WJ, et al. Nanoscale zirconia as a nonmetallic catalyst for graphitization of carbon and growth of single- and multiwall carbon nanotubes. Journal of the American Chemical Society. 2009;131(34):12144-12154.

26. Torres-Torres D, Torres-Torres C, Vega-Becerra O, CheangWong JC, Rodríguez-Fernández L, Crespo-Sosa A, et al. Structured strengthening by two-wave optical ablation in silica with gold nanoparticles. Optics \& Laser Technology. 2015;75:115-122.

27. Boyd RW. Nonlinear Optics. San Diego: Academic Press; 1992.

28. Athalin H, Lefrant S. Correlated method for quantifying mixed and dispersed carbon nanotubes: analysis of the Raman band intensities and evidence of wavenumber shift. Journal of Raman Spectroscopy. 2005;36(5):400-408.

29. Gohier A, Ewels CP, Minea TM, Djouadi MA. Carbon nanotube growth mechanism switches from tip- to basegrowth with decreasing catalyst particle size. Carbon. 2008;46(10):1331-1338.
30. Kumar M, Ando Y. Chemical vapor deposition of carbon nanotubes: a review on growth mechanism and mass production. Journal of Nanoscience and Nanotechnology. 2010;10(6):3739-3758.

31. Busch K, von Freymann G, Linden S, Mingaleev SF, Tkeshelashvili L, Wegener M. Periodic nanostructures for photonics. Physics Reports. 2007;444(3-6):101-202.

32. Dal Negro L, Feng NN, Gopinath A. Electromagnetic coupling and plasmon localization in deterministic aperiodic arrays. Journal of Optics A: Pure and Applied Optics. 2008;10(6):064013.

33. Rozhin AG, Sakakibara Y, Kataura H, Matsuzaki S, Ishida K, Achiba Y, et al. Anisotropic saturable absorption of single-wall carbon nanotubes aligned in polyvinyl alcohol. Chemical Physics Letters. 2005;405(4-6):288-293.

34. Torres-Torres C, Mercado-Zúñiga C, Martínez-González CL, Martínez-Gutiérrez H, Rebollo NR, Trejo-Valdez M, et al. Optical Kerr effect exhibited by carbon nanotubes and carbon/metal nanohybrid materials. Physica E: Low Dimensional Systems and Nanostructures. 2015;73:156-162.

35. Kamaraju N, Kumar S, Sood AK, Guha S, Krishnamurthy S, Rao CNR. Large nonlinear absorption and refraction coefficients of carbon nanotubes estimated from femtosecond z-scan measurements. Applied Physics Letters. 2007;91:251103.

36. Izard N, Billaud P, Riehl D, Anglaret E. Influence of structure on the optical limiting properties of nanotubes. Optics Letters. 2005;30(12):1509-1511. 